\title{
Pêndulo físico amortecido por atrito seco
}

\author{
Physical Pendulum Damped by Dry Friction
}

\author{
Graziele Bezerra Santos ${ }^{1}$, Diego de Assis Santos ${ }^{1}$, Thiago de Oliveira Coghi ${ }^{1}$, José Martins de \\ Oliveira Jr. ${ }^{1}$, Norberto Aranha ${ }^{1}$, Waldemar Bonventi Jr. $\left.{ }^{*}\right]$ \\ ${ }^{1}$ Universidade de Sorocaba, Programa de Pós-Graduação em Processos Tecnológicos e Ambientais, Sorocaba, SP, Brasil.
}

Recebido em 01 de abril de 2020. Revisado em 20 de abril de 2020. Aceito em 14 de maio de 2020.

\begin{abstract}
Neste trabalho explora-se o movimento pendular amortecido por atrito seco. O atrito seco (ou coulombiano) difere do atrito viscoso pelas seguintes características: a) a amplitude de oscilação decai linearmente, enquanto que o atrito viscoso resulta em decaimento exponencial; b) a frequência do movimento é igual ao pêndulo não amortecido, sendo que, no amortecimento viscoso, a frequência "natural" de oscilação diminui com o fator de amortecimento. Foram realizados experimentos com uma ripa de madeira suspensa oscilando em contato lateral com uma placa lisa e um DVD oscilando suspenso pela borda, onde o atrito estava no eixo que o suspendia. Os movimentos foram gravados e analisados com o software Tracker. Foi possível obter os parâmetros de decaimento e a força de atrito em cada caso. Foi também calculada a força de arrasto devida ao ar, mostrando que esta era desprezível em relação à força de atrito seco.
\end{abstract}

Palavras-chave: Pêndulo físico, Atrito Seco, Software Tracker.

In this work, pendular movement damped by dry friction was studied. Dry friction differs from viscous friction by: a) the amplitude of oscillation linearly decays, whereas in the viscous damping the oscillation decays exponentially; b) the frequency of oscillation is keeping in presence of dry friction, however, the frequency of oscillation is reduced due to damping factor in viscous case. Some experiments were performed using a wood clapboard suspended and oscillating in lateral contact with a flat plate and using a DVD suspended by an axis stuck on its edge, where the friction was in this axis. The movements were recorded an analyzed with aid of software Tracker. It was possible to obtain decay parameters and friction force in both cases. Also, viscous damping forces were calculated due to drag from the air and showed to be negligible with respect to dry friction. Keywords: Physical pendulum, Dry Friction, Tracker Software.

Keywords: Physical pendulum, Dry Friction, Tracker Software.

\section{Introdução}

O movimento oscilatório é um dos principais tópicos da Física a serem abordados, desde o ponto de vista educacional até as aplicações em engenharia. Logo, a compreensão precisa deste movimento é de importância vital, não só para os físicos, mas também para estudantes de engenharia. O movimento oscilatório real envolve não apenas a dinâmica do movimento em si, relacionada ao corpo que oscila e o sistema (se modelo massa-mola ou pêndulo). Em ambos, dois tipos de amortecimento podem ocorrer: amortecimento viscoso ou amortecimento seco. O estudo do amortecimento viscoso é um tema padrão em todos os livros didáticos de física e também há uma série de trabalhos que lidam com amortecimento viscoso de oscilações mecânicas [1], [2], [3], [4], [5], porém o movimento oscilatório amortecido pelo atrito seco é menos explorado na literatura. Alguns trabalhos neste sentido encontram-se em [6], [7], [8].

O atrito presente no contato entre superfícies é normalmente abordado nos livros textos como um fenômeno

*Endereço de correspondência: waldemar.bonventi@prof.uniso.br que depende da textura da superfície, porém, esta é uma abordagem bem simples sobre o problema. Experimentos indicam uma dependência do atrito de uma grande variedade de parâmetros, incluindo a velocidade de deslizamento, aceleração, distância crítica de deslizamento, temperatura, carga elétrica, força normal, umidade, tipo e preparação da superfície e também a combinação de materiais em contato. Em muitas aplicações de engenharia, o sucesso de modelos preditivos de resultados experimentais continua fortemente sensível ao modelo de atrito [9].

Neste trabalho damos enfoque ao pêndulo físico com predominância do atrito seco sobre o viscoso, em que um corpo rígido oscila suspenso com seu centro de massa abaixo do pivô de suspensão com secção de choque frontal com o ar muito pequena e superfícies lisas. Foram feitas análises relativas ao tipo de oscilação com medidas experimentais. Os dados de posição vs tempo foram obtidos com auxílio do Tracker, um software livre utilizado na análise de dados experimentais de movimentos [10].

Foram realizados dois tipos de experimento. No primeiro, foi utilizada uma ripa similar à utilizada em [5], 
porém, naquela ocasião, o atrito com o pivô era mínimo e a principal contribuição para o amortecimento da oscilação foi da resistência do ar, em que se observou claramente uma oscilação reduzida exponencialmente. Neste trabalho, a ripa suspensa por um orifício em sua extremidade com atrito desprezível no pivô, oscilou encostada a uma parede lisa de modo que as oscilações foram fortemente amortecidas pelo contato constante com a parede, caracterizando atrito seco. No segundo, foi utilizado um DVD, posto a oscilar na direção do plano da superfície circular, suspenso apenas pelo pivô e sem contato lateral algum. O DVD apresenta faces bem lisas e espessura muito pequena, de modo que o arrasto do ar fosse desprezível, ao mesmo tempo em que havia um atrito significativo no pivô. Com isto, foi possível calcular o coeficiente de atrito em um dos casos estudados e a força de arrasto devido ao ar nos dois casos.

\section{Considerações teóricas}

O atrito é a força de resistência ao movimento relativo entre superfícies sólidas, camadas de fluidos e outros materiais que deslizam um contra o outro. O atrito é classificado em função da natureza do material e a forma de contato: o atrito seco ou atrito de Coulomb é a força de oposição ao movimento relativo às faces de duas superfícies sólidas em contato. Este atrito é ainda descrito como atrito estático ("aderência") no caso de superfícies sem movimento relativo e atrito cinético entre superfícies em deslizamento relativo. Sem considerar o atrito no nível atômico ou molecular, o atrito seco geralmente surge da interação envolvendo características da superfície ("asperezas"). Em outra categoria está o atrito fluido, descrito como a força de cisalhamento entre as camadas de um fluido viscoso, as quais se movem uma em relação à outra [11], [12]. Um caso particular deste surge quando um fluido lubrificante separa duas superfícies sólidas [13], [14], [15]. Por outro lado, quando um sólido está imerso em um fluido, surge o atrito viscoso, que é um componente do arrasto, ou seja, a força que resiste ao movimento de um fluido na superfície do corpo. Há também o atrito interno, dado pela força que resiste ao movimento entre os elementos que compõem um material sólido enquanto sofre deformação [14].

$\mathrm{O}$ atrito não é em si uma força fundamental. O atrito seco, objeto deste estudo, surge da combinação de efeitos de adesão entre superfícies, rugosidade, deformação da superfície e contaminações que nela possam ocorrer. A complexidade das interações decorrentes torna impraticável o cálculo do atrito a partir de princípios fundamentais da Física, sendo necessário o uso de métodos empíricos para análise deste fenômeno.

\subsection{Atrito seco}

O modelo mais simples para o atrito seco é dado pela lei de atrito de Coulomb, que ainda assim exige uma série de considerações. As propriedades elementares do atrito cinético foram descobertas experimentalmente entre os séculos XV e XVIII, resultando em três leis empíricas:

- Primeira lei de Amonton: a força de atrito é diretamente proporcional à carga aplicada.

- Segunda Lei de Amonton: A força do atrito é independente da área aparente de contato.

- Lei do atrito de Coulomb: o atrito cinético é independente da velocidade de deslizamento. A lei do atrito de Coulomb é resumida pela simples equação escalar 1.

$$
F=\mu N
$$

em que $\boldsymbol{F}$ é a força de atrito proporcionada pela reação normal de contato $N$ entre as duas superfícies em questão. Ambas estão relacionadas pelo coeficiente de atrito . Desde que $\boldsymbol{F}$ e $\boldsymbol{N}$ sejam expressas na mesma unidade de medida (newton no Sistema Internacional de Unidades ou kgf, por exemplo, o coeficiente de atrito é grandeza adimensional.

Ruina [13] discute o atrito cinético e o estático ao considerar os casos de movimento relativo entre as superfícies em contato, resumindo-o no Quadro 1.

O sentido da força de atrito $\boldsymbol{F}$ em um corpo $A$ está no sentido oposto à velocidade de escorregamento de $A$ em relação a $B$. Pelo princípio da ação e da reação, deduzimos que a força no corpo $B$ está no sentido oposto. Essa força também é oposta à velocidade relativa de escorregamento de $B$ em relação a $A$. Ou seja, $\boldsymbol{F}$ resiste ao movimento relativo entre $A$ e $B$. Na Figura 1 estão esquematizados o sentido de deslocamento (escorregamento - slip rate) $\dot{\delta}$ do corpo, a atuação da força $\boldsymbol{F}$ de atrito e da reação normal de contato $\boldsymbol{N}$ e um gráfico em que a relação entre estas forças é proporcional ao coeficiente de atrito estático $\mu_{\mathrm{s}}$ (sem escorregamento) ou dinâmico (ou cinético) $\mu_{\mathrm{d}}$, quando há escorregamento $\dot{\delta}$. Note que o coeficiente de atrito estático é maior que o coeficiente de atrito dinâmico. Isto mostra porque é percebido que "um corpo quando começa a escorregar, não pára mais". Se a força que

Quadro 1: atrito "dinâmico"vs. atrito "estático"

\begin{tabular}{|c|c|c|c|}
\hline Escorregamento & $\begin{array}{l}\text { Atrito - interação tangente à } \\
\text { superfície. Opõe-se ao desloca- } \\
\text { mento }(\boldsymbol{v})\end{array}$ & $\vec{F}=-\mu N \frac{\vec{v}}{|\vec{v}|}$ & $\begin{array}{l}\text { Velocidade relativa de escorrega- } \\
\text { mento entre os pontos de contato }\end{array}$ \\
\hline $\begin{array}{l}\text { Contato estacioná- } \\
\text { rio }\end{array}$ & $\begin{array}{l}\text { Componente tangencial da força } \\
\text { de contato }\end{array}$ & $|\vec{F}| \leq \mu N$ & $\begin{array}{l}\text { Limite máximo da componente } \\
\text { tangencial da força de contato }\end{array}$ \\
\hline
\end{tabular}




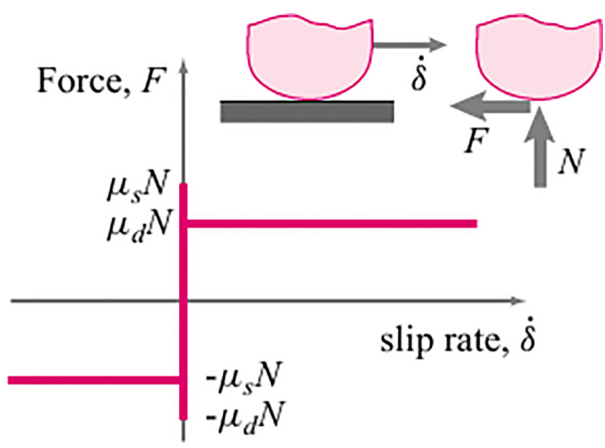

Figura 1: Força de atrito em função do escorregamento $\dot{\delta}$. Fonte: Ruina e Pratap [13]

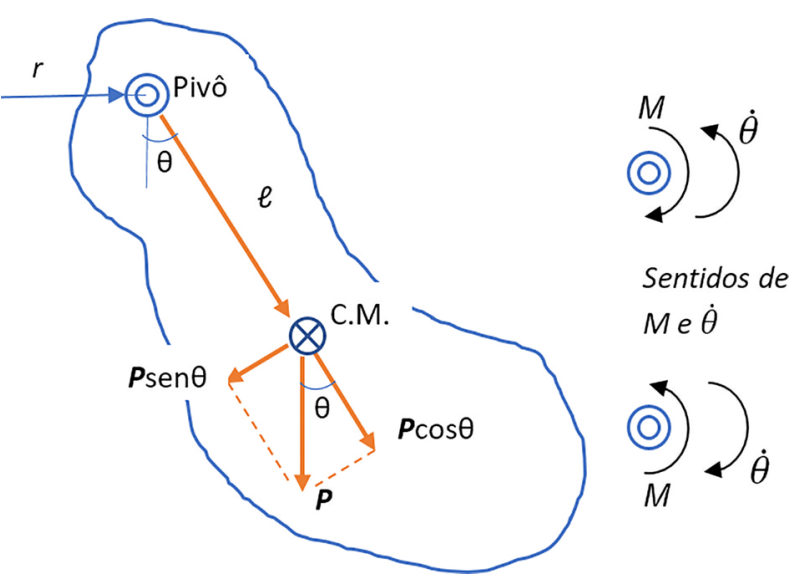

Figura 2: Torque no eixo pivô devido ao atrito seco e forças associadas ao movimento.

iniciou o movimento for maior que a força de atrito estático e for mantida, certamente é maior que a força de atrito dinâmico ou cinético, quando está havendo o escorregamento.

\subsection{Pêndulo físico}

Conforme descrito em [16], [17] e [18], para citar algumas referências, o pêndulo físico é descrito como um corpo rígido suspenso e posto a oscilar verticalmente em torno de um pivô acima do seu centro de gravidade (Figura 2). A equação dinâmica do movimento (eq. 4) apresenta um termo devido ao torque resultante no pivô, $I_{\mathrm{P}} \ddot{\theta}$ provocado pelo torque dado pelo produto entre a componente tangencial do peso $(m g \operatorname{sen} \theta)$ atuando no centro de massa (ou centro de gravidade, aqui ambos coincidem) e a distância $\ell$ do pivô ao centro de massa. $I_{\mathrm{P}}$ é o momento de inércia relativo ao pivô $\mathrm{P}$ e $\sum M \neq 0$ expressa o torque resultante no corpo. Se não forem considerados a resistência do ar (que produz o atrito viscoso) e o atrito seco proporcionado pelo giro do eixo pivô, a única força que gera o movimento pendular é a componente tangencial do peso $m g \ell \cdot \operatorname{sen} \theta$, que se opõe ao sentido de rotação relativo ao pivô.

$$
I_{P} \ddot{\theta}=\sum M=-m g \operatorname{sen} \theta l \rightarrow I_{P} \ddot{\theta}+m g l . \operatorname{sen} \theta=0
$$

Nesta equação $4, I_{\mathrm{P}}$ é o momento de inércia do corpo que oscila pendularmente em relação ao ponto de suspensão (pivô) $\mathrm{P}, \ddot{\theta}=\frac{d^{2} \theta}{d t^{2}}$ é a aceleração angular referente à rotação do corpo em torno do pivô, $m$ é a massa do corpo, $g$ a aceleração gravitacional, $\ell$ é a distância do centro de massa do corpo ao pivô e $\theta$ é o ângulo que a linha entre o pivô e o centro de massa faz com a vertical (prumo). É muito frequente referir-se a $\ell$ como o comprimento do corpo, assim, seu centro de massa distaria $\ell / 2$ do pivô. Estamos considerando que o corpo possui um formato arbitrário e pode ser não homogêneo (Figura 2)

O momento de inércia $I$ é uma medida da inércia rotacional de um corpo que gira em torno de um eixo arbitrário relativo a ele. Esta medida é realizada pela distribuição dos elementos de massa em torno deste eixo, ponderado pelas distâncias a ele. As unidades de medida, no Sistema Internacional (S.I.), são: $\left[I_{\mathrm{P}}\right]=\mathrm{kg} \cdot \mathrm{m}^{2},[\ddot{\theta}]$ $=\mathrm{rad} / \mathrm{s}^{2},[m]=\mathrm{kg},[g]=\mathrm{m} / \mathrm{s}^{2},[\ell]=\mathrm{m}$. Muito importante é lembrar que $\theta$ é medido em radianos $(\mathrm{rad}){ }^{1} \mathrm{em}$ vez de graus $\left(^{\circ}\right)$, este último sendo muito praticado em atividades experimentais. Por conveniência, para corpos relativamente pequenos (da ordem de $0,1 \mathrm{~m}$ ), pode-se expressar o momento de inércia em g.cm².

Para pequenas oscilações, em que $\operatorname{sen} \theta \approx \theta,(\theta \mathrm{em}$ radianos), a equação apresenta a solução

$$
\theta(t)=A \cos (\omega t+\varphi)
$$

a qual, aplicada à eq. 4, resulta na expressão da eq. 6 para a frequência de oscilação "natural" do pêndulo.

$$
\omega=\sqrt{\frac{m g l}{I_{P}}}
$$

bastando, para isso, substituir a (5) em (4). Lembrando que, na equação 4 , deve ser feita a aproximação $\operatorname{sen} \theta \approx \theta$ e calcular $\ddot{\theta}$.

A análise dimensional da equação 6 mostra que $[\omega]$ $=\mathrm{rad} / \mathrm{s}$. Considerando-se esta uma situação ideal, onde não há perda de energia, a amplitude de oscilação permanece constante igual ao ângulo $\theta_{0}$ de onde o pêndulo foi inicialmente abandonado [18].

\subsection{Pêndulo com atrito viscoso}

Bonventi e Aranha [5] realizaram experimento similar com uma ripa suspensa pela extremidade, onde se verificou que o atrito com o eixo pivô na oscilação era desprezível em relação à força de arrasto proporcionada pelo ar (atrito viscoso). Havendo perda de energia por atrito viscoso, atribui-se um termo $b \dot{\theta}$ proporcional à velocidade angular e associado ao torque que desacelera

\footnotetext{
1 O rad também simboliza uma unidade de dose de radiação absorvida, definida como $1 \mathrm{rad}=0,01 \mathrm{~Gy}=0,01 \mathrm{~J} / \mathrm{kg}$, nas unidades CGS como a dose equivalente a 100 ergs de energia absorvidos por um grama de matéria. Não é mais recomendado o uso do sistema CGS.
} 
o movimento. Deste modo, a equação dinâmica torna-se

$$
\begin{array}{r}
I_{P} \ddot{\theta}=\sum M=-m g \operatorname{sen} \theta l-b \dot{\theta} \rightarrow \\
\ddot{\theta}+\frac{b}{I_{p}} \dot{\theta}+\frac{m g l}{I_{P}} \cdot \operatorname{sen} \theta=0
\end{array}
$$

em que $\dot{\theta}=d \theta / d t$, sendo similar às equações tradicionalmente vistas para oscilações lineares amortecidas [16], já $\operatorname{com}$ a aproximação $\operatorname{sen} \theta \approx \theta$. Neste caso, a frequência de oscilação é dependente também do fator de amortecimento $b$ (eq 8).

$$
\omega=\sqrt{\frac{m g l}{I_{P}}-\left(\frac{b}{2 I_{P}}\right)^{2}}
$$

$b$ é um fator de amortecimento, de unidades $\mathrm{kg} \cdot \mathrm{m}^{2} / \mathrm{s}$. Para que o amortecimento não retire toda a energia cinética do sistema antes da primeira oscilação completa, $b<2 \sqrt{m g l I_{\mathrm{P}}}$ (caso subcrítico).

A amplitude de oscilação vai decrescendo exponencialmente, como solução da eq. 7

$$
\theta(t)=\theta_{0} e^{-b t /\left(2 I_{P}\right)} \cos (\omega t+\varphi)
$$

a qual, aplicada à eq. 7 , resulta na expressão da eq. 8 para a frequência de oscilação do pêndulo com amortecimento viscoso [18]. O movimento é oscilatório com amplitude decaindo exponencialmente.

\subsection{Pêndulo com atrito seco}

No caso do pêndulo, onde só há dois pontos de parada nas extremidades do movimento, ocorre um deslizamento com atrito entre o corpo suspenso e o pivô (no experimento do DVD aqui realizado) e entre o corpo suspenso e a superfície lateral (experimento da ripa). A força de atrito seco ou atrito de Coulomb é proporcional à força normal $\boldsymbol{N}$ entre as superfícies deslizantes (eq. 2). No caso em que a perda de energia cinética é consideravelmente maior devido ao atrito do pivô do que a devida à resistência do ar, a equação dinâmica (4) é acrescida do torque resistente $M$ devido ao atrito coulombiano do pivô (ver também o Quadro 1). Observa-se na Figura 2 as grandezas relacionadas à dinâmica do movimento. Analogamente à segunda lei de Newton, considerando rotações, um corpo submetido a uma resultante de torques adquire aceleração rotacional $\ddot{\theta}$ e o torque resistente $M$, cujo sinal depende do sentido de rotação em um dado instante. Nos pêndulos, a componente do peso está sempre exercendo um torque restaurador, analogamente a um sistema massa-mola, em que a mola sempre atua contrária ao sentido do movimento, por isso seu sinal negativo A Figura 2 esquematiza os sentidos dos torques externos Assim, a equação 4 se torna:

$$
I_{P} \ddot{\theta}=-m g \operatorname{sen} \theta l \pm M
$$

e o sinal adequado do torque $M$ provocado pelo atrito seco é convenientemente expresso em termos do sentido de rotação $\dot{\theta} /|\dot{\theta}|$ em torno do pivô (equação 11):

$$
I_{P} \ddot{\theta}+M(\dot{\theta} /|\dot{\theta}|)+m g l . \operatorname{sen} \theta=0
$$

Verifica-se que as dimensões de $I_{P} \ddot{\theta}, M$ e $m g l$ são todas consistentes com as unidades de momento da força ou torque. No S.I., kg.m $\mathrm{m}^{2} / \mathrm{s}^{2}$ ou N.m

Se o pêndulo estiver na posição vertical, a força normal na eq. 1 pode ser substituída pelo peso do corpo rígido, concentrado no seu centro de massa.

Quando o pêndulo sai do prumo, o peso se divide em uma componente tangencial ao movimento $m g \cdot \operatorname{sen} \theta$ e uma componente radial $m g \cdot \cos \theta$ no sentido contrário ao que aponta para o pivô (Figura 2). O atrito no pivô surge como consequência da reação a essa componente radial (a força "normal" nesse caso) vezes o coeficiente de atrito. Assim, o torque $M$ pode ser escrito como

$$
M=F_{\mathrm{AT}} \cdot r=\mu N r=\mu \cdot m g \cdot \cos \theta \cdot r
$$

sendo $r$ o raio do eixo pivô, uma vez que o atrito de escorregamento se dá na borda cilíndrica dele (Figura 2).

No regime de pequenas oscilações, $\operatorname{sen} \theta \sim \theta$ e $\cos \theta \sim 1$. Assim, $M$ é considerado constante durante todo o ciclo $M=\mu . m g . r$ e a solução da eq. 11 é dada por

$$
\theta(t)=A \cos (\omega t+\varphi) \pm M /(m g l)
$$

Substituindo a eq. 13 na 11, conclui-se que a frequência de oscilação é a mesma de um pêndulo físico sem atrito:

$$
\omega^{2}=m g l / I_{\mathrm{P}}
$$

Considerando-se as condições de contorno $\theta(0)=\theta_{0} \mathrm{e}$ $\dot{\theta}_{0}=0$, obtém-se a fase $\varphi=0$ e a amplitude

$$
A=\theta_{0} \mp M /(m g l)
$$

Resta-nos agora considerar como o momento gerado pelo atrito é equacionado. Uma abordagem seria calcular $\theta(t)$ para um ciclo completo de oscilação, mas como o atrito é contrário ao movimento, troca de sinal a cada meio ciclo. Então, realizando este cálculo para meio ciclo (meio período: $T / 2=\pi / \omega)$, de modo que

$$
\theta\left(\frac{\pi}{\omega}\right)=\left(\theta_{0}-\mu r / l\right) \cos \pi+\frac{M}{m g l}=-\theta_{0}+\frac{2 M}{m g l}
$$

de onde se obtém:

$$
M=\frac{m g l}{2}\left(\theta_{0}+\theta\left(\frac{\pi}{\omega}\right)\right)
$$

Assim, no instante inicial, a posição angular do pêndulo é $\theta_{0}$ e, decorrido meio período, o valor dado pela eq. 16. Pela simetria da oscilação, a posição angular em um período completo será $\theta_{0}-4 M / m g \ell$, de onde se infere que, a cada oscilação, a amplitude angular decresce de uma quantidade $4 M / m g \ell$ (constante). Com isto, explicase a envoltória linear do gráfico da posição angular vs. 
tempo (Figura 3), cuja equação de reta é definida pela posição (máxima) a cada ciclo $n$ (eq. 18):

$$
\theta_{\mathrm{MAX}}(n)=\theta_{0}-\frac{4 M}{m g l} \cdot n
$$

Logo, a equação geral para o movimento do pêndulo com atrito coulombiano, dadas as condições de contorno acima mencionadas, é dada por:

$$
\theta(t)=\left(\theta_{0}-\frac{4 M}{m g l} \cdot \frac{t}{T}\right) \cos (\omega t)
$$

É possível determinar experimentalmente o torque redutor proporcionado pelo atrito coulombiano por meio do gráfico da posição angular $\theta$ vs. tempo. Este coeficiente é obtido da inclinação $a=\left[\theta(T)-\theta_{0}\right] / T=(-4 M / m g l) / T$ da reta no gráfico experimental da oscilação (Figura 3 )

Em um dos experimentos aqui realizados, utilizando um DVD como pêndulo, o atrito predominante foi devido ao giro em torno do pivô de suspensão. Nesse caso, o torque redutor das oscilações é obtido pela força de atrito $F_{\text {AT }}$ (no contato do eixo com orifício no qual está inserido) vezes o raio $r$ do eixo (eq. 12). Desta maneira, o coeficiente de atrito entre o eixo e o orifício é obtido pela inclinação $a$ da reta envoltória vista na Figura 3 empregando-se a eq. 19, no que resulta:

$$
a=-4 M /(m g l T)=-4 \mu r /(l T)
$$

Note que a dimensão de $[a]$ no S.I. é s ${ }^{-1}$ e a intensidade do coeficiente de atrito dinâmico é obtida por:

$$
\mu=a T l /(4 r)
$$

No outro experimento, realizado com uma ripa suspensa pelo pivô, mas também em contato com uma parede lisa, o atrito seco considerado é do escorregamento da ripa contra a parede enquanto a ripa oscila suspensa. O atrito com o pivô foi dado como desprezível (ver seção Resultados e referência [5]), tanto que o número de oscilações da ripa até parar é muito menor do que o DVD e nos experimentos de [5].

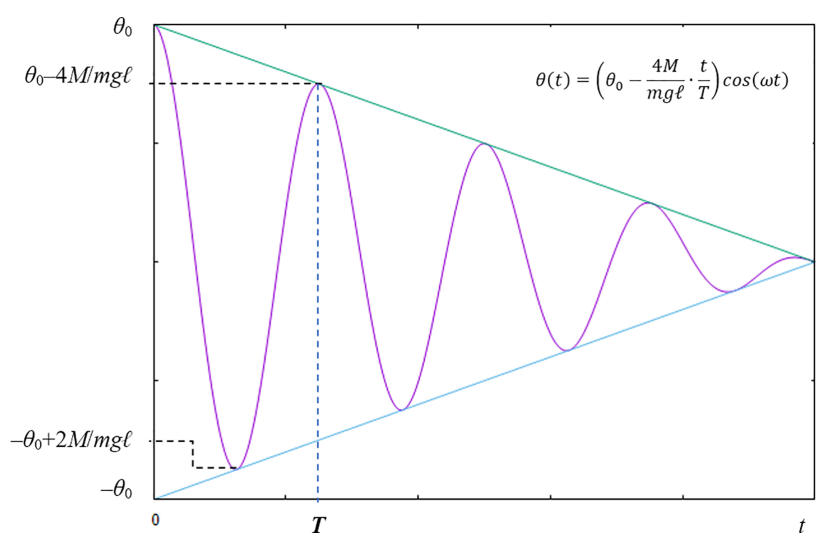

Figura 3: Modelo gráfico da oscilação amortecida por atrito seco
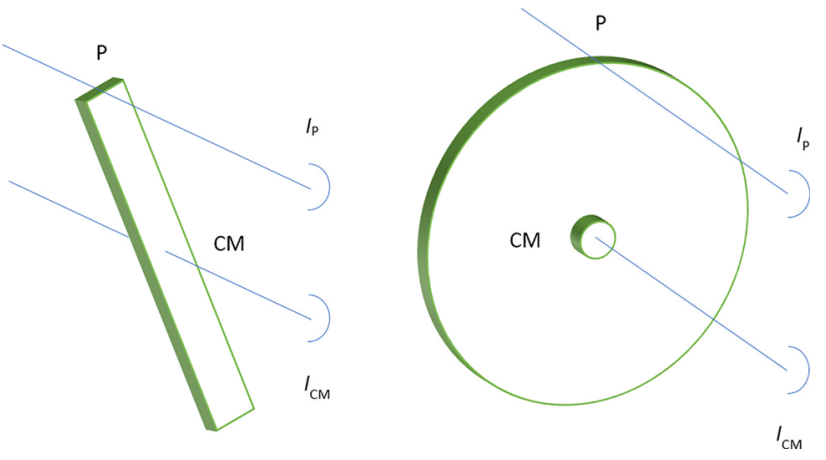

Figura 4: Ilustração dos eixos associados ao momento de inércia I, passando pelo pivô $\mathrm{P}$ e pelo centro de massa CM de cada corpo

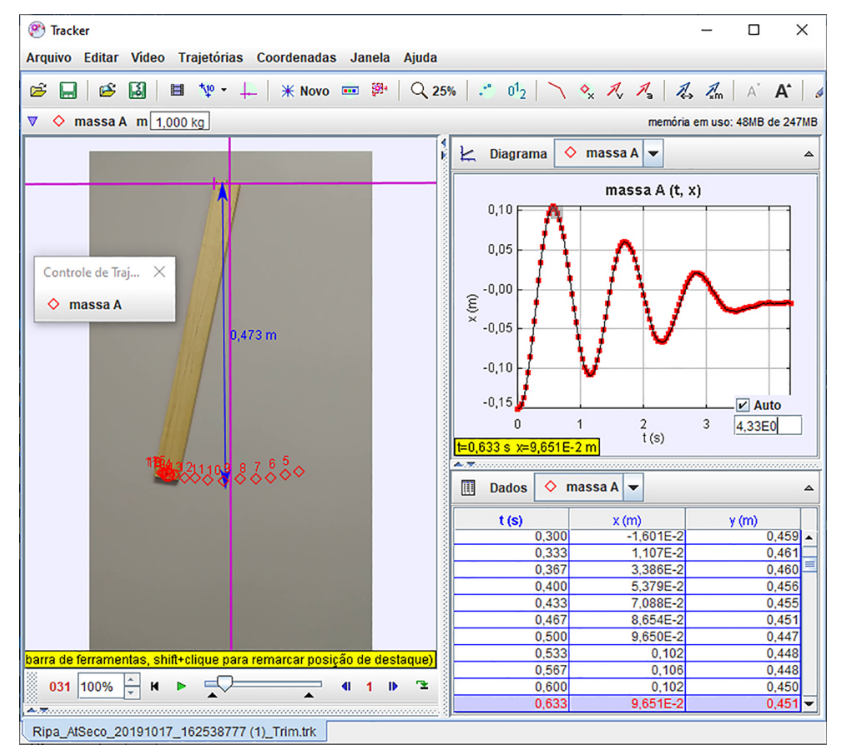

Figura 5: Ripa suspensa por pivô encostada em parede lisa e dados no Tracker

Neste caso, as equações 20 e 21 não se aplicam, mas o modelo geral das equações 13 e 19 é mantido, desde que se consiga medir o torque resultante devido ao atrito da ripa com a parede, em que seções transversais dela realizam movimento circular em torno do pivô e a força de atrito em cada seção é tangencial a esse movimento circular (Figura 4 e Figura 5).

Uma revisão sobre os diversos modelos de atrito desenvolvidos, especialmente os do tipo "seco", foi realizado por Grego [19]. O modelo de atrito seco de Coulomb atende aos propósitos do experimento realizado. Um modelo pode ser mais "atual" ou mais sofisticado em sua formulação, como o de sticktion (static+friction) e seus aperfeiçoamentos teóricos [13]. Entende-se que neste trabalho é desnecessário considerar um modelo mais sofisticado, porque a velocidade é nula apenas nas "extremidades" do movimento (prevalência do atrito estático sobre o dinâmico) e, nestas extremidades, a componente da força peso que acelera o pêndulo invertendo seu movi- 
mento é máxima $(m g \cdot \operatorname{sen} \theta)$, bem maior do que a força de atrito estático (ver seção de Resultados).

Conforme mencionado na introdução, foram realizados dois experimentos: uma ripa de madeira e um disco DVD utilizados como pêndulos. Apresenta-se a seguir o cálculo dos momentos de inércia destes dois corpos em relação ao pivô pelo qual foram suspensos.

\subsection{Momentos de inércia}

Nesta seção apresenta-se o cálculo do momento de inércia, levando-se em conta as características geométricas dos corpos utilizados nos experimentos.

Na Figura 4 estão esquematizados os eixos considerados para este cálculo em cada corpo. $I_{\mathrm{CM}}$ é o momento de inércia dado a partir de um eixo que passa pelo centro de massa (CM) de um corpo. Normalmente, este valor é tabelado em função das dimensões e da geometria do corpo.

Neste trabalho, devido ao movimento oscilatório pendular, consideramos $I_{\mathrm{CM}}$ em um eixo perpendicular ao maior plano dos corpos. $I_{\mathrm{P}}$ é o momento de inércia relativo a um eixo passando pelo pivô, estando paralelo ao eixo de $I_{\mathrm{CM}}$. Sendo assim, $I_{\mathrm{P}}$ pode ser calculado a partir de $I_{\mathrm{CM}}$ fazendo uso do Teorema dos Eixos Paralelos (Teorema de Steiner) [14], [16], [17].

\subsubsection{Momento de inércia de uma placa retangular}

O momento de inércia de uma placa retangular (que representa a ripa utilizada no segundo experimento) relativo a um eixo perpendicular à sua maior superfície (ou seja, na direção de sua espessura), passando pelo seu centro de massa é dado por

$$
I_{\mathrm{CM}}=m\left(h^{2}+w^{2}\right) / 12
$$

em que $h$ é a altura (distância entre bordas opostas) e $w$ é a largura da placa (distância entre as outras bordas opostas). Utilizamos esta nomenclatura devido às dimensões da ripa utilizada, cuja geometria é um retângulo bem alongado.

Levando em conta que o pivô está na extremidade da dimensão mais longa $(h)$ e distante $h / 2$ do centro de massa, aplica-se o Teorema dos Eixos Paralelos para reavaliar o momento de inércia $I_{\mathrm{P}}$ relativo ao novo eixo de oscilação:

$$
I_{\mathrm{P}}=I_{\mathrm{CM}}+m l^{2}
$$

onde $\ell$ é a distância entre o eixo que passa pelo centro de massa (CM) e o novo eixo definido pelo pivô (paralelo àquele), posicionado na extremidade da ripa. Aplicandose esse teorema, o momento de inércia é expresso como

$$
I_{\mathrm{P}}=m\left(h^{2}+w^{2}\right) / 12+m(h / 2)^{2}=m\left(4 h^{2}+w^{2}\right) / 12
$$

Substituindo-se esta equação 24 na eq. 14, obtém-se

$$
\omega_{0}^{2}=6 g h /\left(4 h^{2}+w^{2}\right)=6 g /\left(4 h+w^{2} / h\right)
$$

mostrando que, a frequência de oscilação depende mais fortemente da dimensão $h$ para $h » w$.

\subsubsection{Momento de inércia de um disco}

O momento de inércia de um disco com orifício circular concêntrico (ou anel), relativo a um eixo perpendicular ao seu plano e que passa pelo seu centro de massa, é dado por

$$
I_{\mathrm{CM}}=m\left(R_{1}^{2}+R_{2}^{2}\right) / 2
$$

em que $R_{1}$ e $R_{2}$ são os raios externo e interno do DVD e $m$ sua massa. Aplicando-se o Teorema dos Eixos Paralelos, o momento de inércia torna-se

$$
I_{\mathrm{P}}=m\left(R_{1}^{2}+R_{2}^{2}\right) / 2+m R_{1}^{2}=m\left(3 R_{1}^{2}+R_{2}^{2}\right) / 2
$$

Substituindo-se esta equação 27 na eq. 14, obtém-se

$$
\omega^{2}=2 g l /\left(3 R_{1}^{2}+R_{2}^{2}\right)
$$

o que mostra claramente que a frequência natural de oscilação harmônica do pêndulo físico independe da sua massa.

\subsection{Aceleração gravitacional g corrigida}

O valor da aceleração gravitacional, necessário para calcular a frequência de oscilação relacionada ao momento de inércia de um pêndulo físico, geralmente é utilizado como $g=9,8 \mathrm{~m} / \mathrm{s}^{2}$. Conforme discussão em [5], procurou-se um valor mais preciso para a localidade e altitude onde o experimento foi realizado. Adotou-se o cálculo dado por Mezzalira et al [20]

$$
\begin{aligned}
& g=9,7803184 \cdot\left[1+0,0053024 \cdot \operatorname{sen}^{2}(L)\right. \\
& \left.-0,0000059 \cdot \operatorname{sen}^{2}(2 L)\right]-3,086 \cdot 10^{-6} H
\end{aligned}
$$

em que $L$ é a latitude e $H$ a altitude do local. Adotandose a altitude do local de realização dos experimentos como $600 \mathrm{~m}$ acima do nível do mar e latitude de $23^{\circ} 30^{\prime}$ [21], substitui-se estes valores na eq. 29 , obtendo-se a aceleração da gravidade igual a $9,78668 \mathrm{~m} / \mathrm{s}^{2}$. Pelos desvios relativos tipicamente obtidos nas medidas neste trabalho, adotou-se $g=9,79 \mathrm{~m} / \mathrm{s}^{2}$.

\section{Materiais e Métodos}

Neste estudo foram utilizados como pêndulo:

1. uma ripa de eucalipto de massa $m=(68,14 \pm 0,01)$ g, medida com uma balança semianalítica, comprimento $h=(473,0 \pm 0,5) \mathrm{mm}$, largura $w=(41,0 \pm 0,5)$ $\mathrm{mm}$, espessura $b=(10,0 \pm 0,5) \mathrm{mm}$, medidos com uma trena;

2. um DVD de massa $m=(15,00 \pm 0,01) \mathrm{g}$, medida com uma balança semianalítica, diâmetro externo $\mathrm{D}_{1}=(120,0 \pm 0,5) \mathrm{mm}$, diâmetro do orifício $\mathrm{D}_{2}=$ $(15,0 \pm 0,5) \mathrm{mm}$ e espessura $e=(2,0 \pm 0,2) \mathrm{mm}$, medidos com um paquímetro. 
Para que fosse possível o estudo do atrito seco, a ripa foi suspensa por um pequeno orifício feito bem próximo à sua extremidade $(2 \mathrm{~mm})$, cujo diâmetro também foi de $2 \mathrm{~mm}$, feito cuidadosamente com uma broca para madeira. A ripa foi posta a oscilar encostada em uma parede lisa constituída de placas para divisórias, havendo assim atrito de escorregamento entre a face da ripa e a superfície da parede, resultando em poucas oscilações devido ao forte amortecimento resultante (Figura 5).

O centro de massa da ripa foi determinado pelo método do prumo, da mesma maneira descrita em [5]. A distância entre o furo central (eixo de oscilação) e o centro de massa da ripa foi medida com uma régua e o valor obtido foi $\ell=(234,0 \pm 0,5) \mathrm{mm}$. A ripa foi posta a oscilar a partir de um angulo de aproximadamente $\arctan (0,151 / 0,473)$ $=17,7^{\circ}$ em relação à vertical (corresponde a $0,309 \mathrm{rad}$, com $\operatorname{sen}(0,309)=0,304$, diferença de $1,6 \%$

O movimento pendular da ripa foi medido com o auxílio do Tracker, usando como referência um dos orifícios feito na extremidade inferior após a ripa estar suspensa pelo pivô. O "Tracker" é um software livre que trabalha com a linguagem Java para análise e modelagem de movimentos gravados em vídeo [10]. Ele tem sido proposto como alternativa para a coleta e análise de dados experimentais de movimentos.

O movimento do DVD foi medido suspendendo por uma fita adesiva presa na borda deste, na qual deixou-se uma sobra para fixação no pivô. O eixo pivô e o DVD estão rigidamente ligados e o eixo atravessa um orifício feito em um suporte de plástico escuro, de modo a girar com um certo atrito, mas mantendo o eixo horizontal. Com isto, o pivô ficou rente à borda do DVD e a distância medida do pivô ao centro de massa foi $\ell=(121,0 \pm 0,5)$ $\mathrm{mm}$ (Figura 6). A massa da fita adesiva (de largura 25 $\mathrm{mm})$ é de $(0,20 \pm 0,01) \mathrm{g}$ para cada $10 \mathrm{~cm}$ de comprimento, medida com uma balança semianalítica. Como estava junto ao pivô para fixação, sua massa e momento de inércia foram desconsiderados. $\mathrm{O}$ conjunto teve uma folha em branco suspensa por trás que sustentava uma régua na sua extremidade inferior, para fins de calibração de escala. O DVD foi posto a oscilar a partir de um angulo de $20,0^{\circ}$ em relação à vertical. Para medir o ângulo inicial de inclinação $\theta_{0}$ do DVD em relação a vertical foi utilizado o

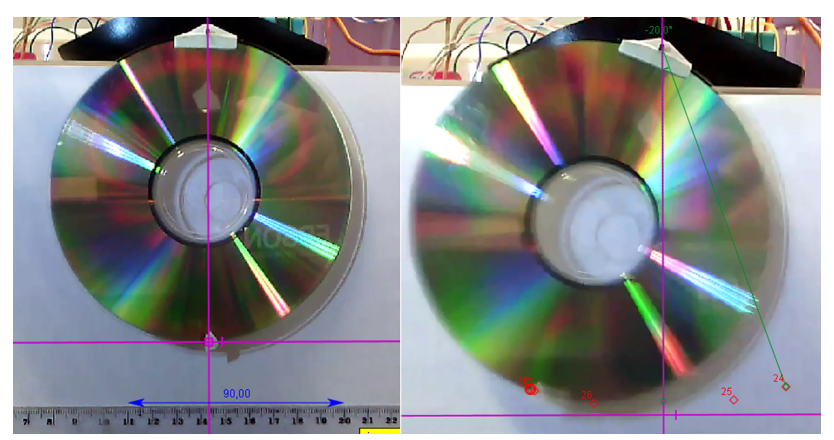

Figura 6: Montagem experimental do DVD e coleta de dados para o Tracker próprio transferidor do "Tracker", indicado na Figura 6 à direita. Sobre a harmonicidade do movimento oscilatório, $20,0^{\circ}=0,349 \mathrm{rad}$ e $|\operatorname{sen}(0,349)-0,349|=0,007$. Além disso, um pequeno ponto foi marcado na posição mais inferior da borda do DVD para seguir seu movimento com o Tracker.

A tomada das posições do pêndulo durante sua oscilação foi realizada inicialmente com o posicionamento do eixo de referência e da escala (em lilás e azul, respectivamente) - ver Figura 5 e Figura 6, calibrados com auxílio da imagem da régua. Em seguida, foram marcadas as posições do ponto mais inferior do corpo em cada frame do vídeo. Estas marcações consistem em "clicks" com o "mouse" na imagem sobre o ponto escolhido que percorre sua trajetória durante a oscilação. Foram tomadas as posições na direção horizontal $(x)$, usando como origem das coordenadas aquelas definidas pelos eixos de cor lilás das Figuras 5 e 6.

A diferença entre a configuração das medidas para o Tracker na ripa (Figura 5) e o DVD (Figura 6) foi que bastou a calibração do Tracker pelo comprimento da ripa (medida antes da montagem experimental) para determinação da escala de movimento em relação ao vídeo, para fins de medidas da amplitude de oscilação.

Como um dos objetivos do experimento era utilizar configurações e equipamentos acessíveis, utilizou-se para a filmagem do movimento um smartphone, com câmera de resolução $5 \mathrm{MP}$, tomada a uma distância aproximada de $20 \mathrm{~cm}$ do conjunto. A duração da filmagem foi de aproximadamente $15 \mathrm{~s}$, gerando 180 quadros com dados de tempo e posições nas direções $x$ e $y$. A oscilação da ripa inicia no quadro $\mathrm{n}^{\circ} 12(t=0,0 \mathrm{~s})$, quando foi liberada e termina no quadro $156(t=4,3 \mathrm{~s})$. A oscilação do DVD inicia-se no quadro $\mathrm{n}^{\circ} 22(t=1,9 \mathrm{~s})$, quando o mesmo foi liberado e termina no quadro $150(t=12,3 \mathrm{~s})$.

\section{Resultados e Discussão}

De posse dos dados experimentais efetuou-se a análise dos resultados fornecidos pelo "Tracker". O gráfico da oscilação resultante da ripa, gerado no próprio Tracker, pode ser observado na Figura 7, juntamente com o modelo de função oscilatória amortecida por atrito seco ajustada sobre os dados experimentais (linha sólida). Observa-se neste gráfico que a última oscilação prevista em teoria (linha azul) não ocorre (pontos experimentais) devido a uma desaceleração mais forte no final do movimento (provavelmente alguma característica ligeiramente diferente no contato entre as superfícies naquele local, alterando a força de reação normal de contato). Ainda assim, esta particularidade não interfere na obtenção dos parâmetros do movimento.

Da mesma maneira, o gráfico da oscilação do DVD pode ser observado na Figura 8, com o modelo de função oscilatória ajustada. Observa-se que, com o decaimento linear dos máximos de cada oscilação, esta deveria cessar em cerca de 10,2 s, no entanto, a partir de 9,2 s há 
alteração no modo de amortecimento (observando-se a envoltória exponencial ajustada, no destaque superior da Figura 8). Na seção seguinte, foram parametrizados os dois regimes de movimento, antes e depois de $9,2 \mathrm{~s}$ para análise e discussão.

\subsection{Ajuste dos parâmetros}

Para ajuste da função dada na (19), utilizou-se a janela "DataTool" do Tracker. Com a opção "autofit" habilitada, informa-se a margem de erro admissível para cada para metro $(10 \%, 1 \%$ ou $0,1 \%)$ para a otimização automática deles. Frequentemente eì necessário informar os valores aproximados dos parâmetros iniciais para que o programa inicie o processo de ajuste de modo a possibilitar sua convergência. No caso do ajuste utilizando o "Tracker", basta "clicar" diretamente no gráfico com o botão direito do "mouse" e, no menu flutuante, selecionar a opção "Analisar". É interessante iniciar um ajuste mais grosseiro escolhendo-se erro de $10 \%$ no programa de ajuste e depois ir refinando para $1 \%$ e finalmente $0,1 \%$. Tendo procedido desta maneira, o ajuste ficou bem preciso, observando-se o erro quadrático médio calculado em tempo real pelo Tracker.

A função ajustada segue o modelo de oscilação harmônica mostrado na eq. 19, porém $\omega=2 \pi f$ foi escrito explicitamente para fins de ajuste experimental a partir da frequência de oscilação. Também, o tempo foi deslo-

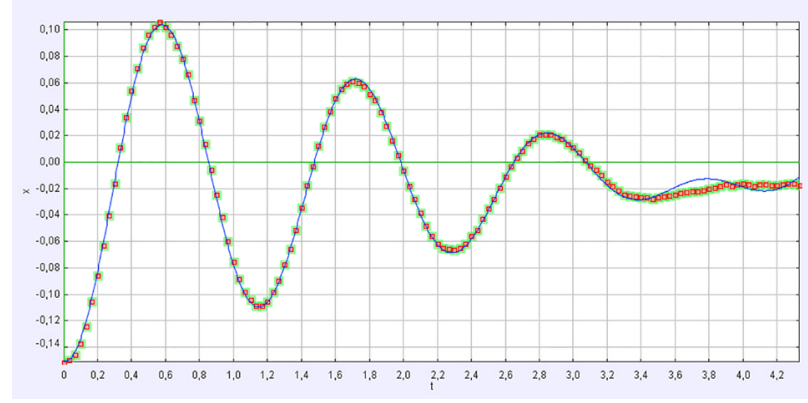

Figura 7: Dados experimentais e função ajustada da oscilação da ripa

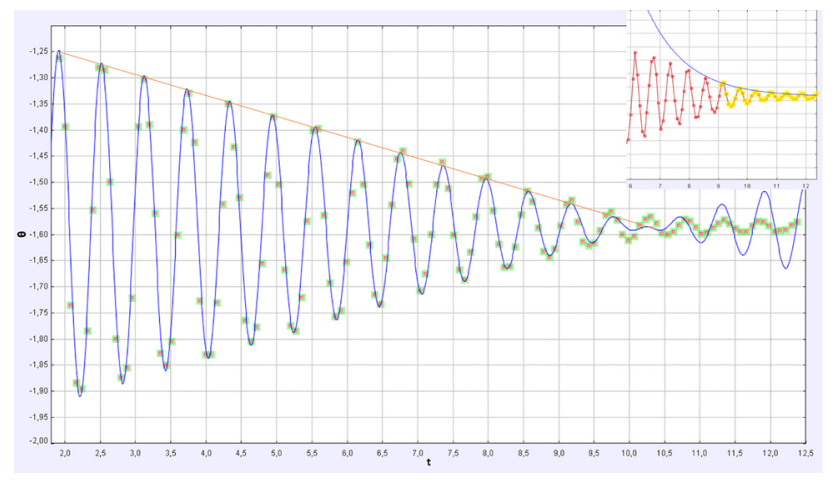

Figura 8: Dados experimentais e funções ajustadas na oscilação do DVD. No detalhe superior, envoltória com decaimento exponencial no final do movimento. cado de $t_{0}$ pelo fato de que a oscilação começou depois do início da gravação (no caso do DVD - Figura 8) e a envoltória expressando a amplitude decaindo linearmente foi reescrita como $\left(\theta_{0}-\frac{4 M}{m g l} \cdot \frac{t}{T}\right)=A\left[1-B\left(t-t_{0}\right)\right]$, obtendo-se a função horária dada em (30). Observou-se que o parâmetro mais crítico de ajuste foi a frequência, seguido da constante de base $C$ e do fator de decaimento $B$, nesta ordem de importância.

$$
\theta(t)=A\left(1-B\left(t-t_{0}\right)\right) \cos \left(2 \pi f\left(t t_{0}\right)\right)+C
$$

O termo $1-B\left(t-t_{0}\right)$ parametriza a reta envoltória da curva de oscilação cossenoide (Figura 8). Ainda, $t_{0}$ assinala o início do movimento em relação à filmagem e o parâmetro $C$ é a posição da linha de base dos pontos.

Para a ripa, os parâmetros de ajuste seguem na função horária:

$$
\begin{aligned}
\theta(t)= & -0,1375 .(1-0,2615(t-0,0162)) \\
& \times \cos (2 \pi .0,8732 .(t 0,0162))-0,0132
\end{aligned}
$$

Para o DVD, inserindo a função de ajuste e valores iniciais para os parâmetros, a expressão fornecida pelo "Tracker" após a convergência dos valores foi

$$
\begin{aligned}
\theta(t)= & 0,3378 .(1-0,120 .(t-1,914)) \\
& \times \cos (2 \pi .1,651 .(t 1,914))-1,585
\end{aligned}
$$

Este ajuste teve como requisito o desvio de $1 \%$ permitido em cada um dos parâmetros $A, B, C, t_{0}$ e $f$ para resultar no desvio quadrático mencionado.

Neste interim, não há o que comparar nos dois movimentos, pois são corpos de conformações bem distintas como também as forças de atrito seco nos dois casos tiveram origens diferentes: no caso da ripa foi o contato da parede ao longo do corpo, cuja reação normal $\vec{N}$ não pôde ser medida. No DVD foi devido ao eixo pivô.

\subsection{Momentos de inércia}

O momento de inércia calculado para o modelo estático a partir da equação 24 fornece o seguinte resultado para a ripa

$$
\begin{aligned}
I_{\mathrm{P}} & =68,14 \cdot\left(4 \cdot 47,3^{2}+4,1^{2}\right) / 12 \\
& =50912 \mathrm{~g} \cdot \mathrm{cm}^{2}=5,09 \cdot 10^{-3} \mathrm{~kg} \cdot \mathrm{m}^{2}
\end{aligned}
$$

E pela oscilação observada, a partir da eq. 14,

$$
\begin{aligned}
I_{\mathrm{EXP}} & =\frac{m g l}{\omega^{2}}=0,06814.9,8 \cdot(0,473 / 2) /(2 \pi \cdot 0,8732)^{2} \\
& =5,25 \cdot 10^{-3} \mathrm{~kg} \cdot \mathrm{m}^{2}
\end{aligned}
$$

Resultando em um erro relativo entre os dois valores de $3,1 \%$, o que parece ser bem razoável nas condições experimentais apresentadas.

Para o DVD (eq. 27)

$$
\begin{aligned}
I_{\mathrm{P}} & =0,015\left(3 \cdot 0,06^{2}+0,075^{2}\right) / 2=8,14 \cdot 10^{-5} \mathrm{~kg} \cdot \mathrm{m}^{2} \\
& =814 \mathrm{~g} \cdot \mathrm{cm}^{2}
\end{aligned}
$$


e, utilizando a eq. 14, chegamos ao momento de inércia do DVD

$$
\begin{aligned}
I_{\mathrm{EXP}} & =m g l / \omega^{2}=0,015.9,79 \cdot 0,06 / 107,79 \\
& =8,18 \cdot 10^{-5} \mathrm{~kg} \cdot \mathrm{m}^{2}=818 \mathrm{~g} \cdot \mathrm{cm}^{2}
\end{aligned}
$$

Comparando-se ambos os resultados, observa-se um erro relativo entre eles de $0,5 \%$.

Este erro é menor se comparado com os resultados da literatura. Em [22] foi realizada uma montagem com sensor oìptico para o estudo do movimento de um disco rígido. Neste trabalho o momento de inércia foi obtido indiretamente através da corrente elétrica do sistema de detecção eletrônico, resultando em um erro menor que $5 \%$ entre o momento de inércia teórico e o experimental. Eadkhong et al [23] utilizaram o "Tracker" no estudo do momento de inércia de um cilindro, obtendo erros na faixa de $0,4 \%$ a 3,3\%. Bonagamba e colegas [24] também utilizaram um sistema de detecção óptico com sensor e receptor infravermelhos, no estudo de oscilação de corpos rígidos e obtiveram erros relativos que variaram de $1,4 \%$ a $3,4 \%$.

\subsection{Obtenção do coeficiente de atrito dinâmico}

Nesta seção, os coeficientes de atrito são obtidos a partir dos dados experimentais vistos nos gráficos e das funções ajustadas.

\subsubsection{Pelo contato da ripa com a parede}

Conforme descrito na configuração experimental, a ripa oscilava encostada a uma parede lisa de modo a manter um contato constante ao longo do movimento, que era diminuído pelo atrito entre a ripa e a parede.

Os coeficientes angulares das retas (superior e inferior) que passam pelos máximos (e mínimos, respectivamente) das oscilações ajustadas no Tracker forneceram os valores $a=-0,0372$ (superior, decrescente) e $a=0,0368$, resultando em uma diferença de 1,1\%. Adotando-se o valor médio entre as duas retas ajustadas $(0,0370)$ e levando em conta a expressão que relaciona o torque amortecedor com a variação da amplitude máxima (eq. 20),

$$
\begin{aligned}
a & =(-4 M / m g l) / T \longrightarrow 0,0370 \\
& =[4 M /(0,068.9,79.0,234)] / 1,145 \\
& \longrightarrow M=1,67.10^{-3} \text { N.m }
\end{aligned}
$$

Este torque é causado pelo contato da face posterior da ripa escorregando pela parede, sendo que a porção da ripa no nível do pivô não escorrega, somente gira em torno dele. As seções transversais da ripa descrevem movimentos circulares, com a extremidade inferior movendo-se com velocidade tangencial $\theta(t) . h=\theta(t) .2 l$. A seção média da ripa (na altura de seu C.M.) também descreve um movimento circular e desloca-se com velocidade tangencial $\theta(t) . h / 2=\theta(t) . l$; e assim por diante. Estas velocidades são proporcionais à medida em que tais seções transversais se afastam do pivô até sua extremidade inferior.

No entanto, e este é um ponto essencial em nossas considerações, o modelo de atrito seco adotado é independente da velocidade. Assim, é assumido que o torque obtido acima provém de uma força de atrito "média" ao longo da ripa, ou seja,

$$
F_{\mathrm{AT}}=\frac{M}{2 \mathrm{l}}=\frac{1,67 \cdot 10^{-3}}{0,473}=3,5 \cdot 10^{-3} \mathrm{~N}
$$

Nesse caso, não há como calcular o coeficiente de atrito entre a ripa e a parede pois não se sabe o valor da força normal de contato entre elas.

Mesmo o torque e a força de atrito sendo muito pequenos (da ordem de $10^{-3}$ ), têm a magnitude suficiente para que ocorram no máximo as três oscilações observadas na Figura 7.

Sendo o atrito de arrasto aerodinâmico da ripa calculada pela secção transversal retangular do seu perfil $h . b=(0,473.0,010) \mathrm{m}^{2}$, considerando que a extremidade presa ao pivô não se desloca na direção transversal e a extremidade inferior se desloca à velocidade tangencial $v=\omega(t) \cdot h$, a força de arrasto (devida somente à secção de choque definida pelo perfil da ripa, veja [25]) pode ser calculada como

$$
F_{\mathrm{ARR}}=-C_{x} \rho v^{2} A / 2
$$

Em que

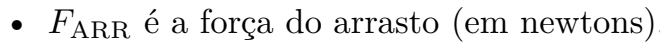

- $C_{x}$ é o coeficiente de arrasto (determinado experimentalmente, adimensional). Para uma placa retangular, é dado aproximadamente como 1,3

- $\rho$ é a massa específica do fluido. Para o ar, tem o valor de $1,20 \mathrm{~kg} / \mathrm{m}^{3}$ a $20^{\circ} \mathrm{C}$ e 1 atmosfera,

- $A$ é a área de referência. Adotamos a área frontal (ao movimento) $h . b=(0,473.0,010) \mathrm{m}^{2}$

- $v$ é a velocidade do objeto em relação ao fluido $(\mathrm{m} / \mathrm{s})$.

A Figura 9 ilustra o efeito do arrasto aerodinâmico nos perfis dos corpos estudados. Note que, no DVD, os trechos de área considerados para o arrasto nem sempre estão perpendiculares ao movimento circular realizado em torno do pivô. Por isso, aproximando-se da extremidade inferior o arrasto frontal se torna praticamente nulo.

A velocidade do perfil na extremidade da ripa, com medição feita pela ferramenta do Tracker "medir declive" (na janela de análise de dados), foi obtida como valendo 0,641 m/s, no ponto mais baixo da trajetória (quarto de período de oscilação), na primeira oscilação, que é a maior velocidade que o pêndulo pode atingir. Isto permite um cálculo estimativo da força de arrasto máxima, já que a mesma varia com a velocidade de oscilação do corpo. A força de arrasto é calculada integrando-se as velocidades ao longo do comprimento da ripa e obtendo-se sua média 
em um período de oscilação. A velocidade média ao longo da ripa, como função do tempo é calculada na eq. 34 .

$$
\begin{aligned}
\overline{v(t)}= & \frac{1}{2 \mathrm{l}} \int_{y=0}^{2 \mathrm{l}} v(t, y) d y=\frac{1}{2 \mathrm{l}} \int_{y=0}^{2 \mathrm{l}} \theta^{\prime}(t) \cdot y d y \\
& =\frac{1}{2 \mathrm{l}} \theta^{\prime}(t) \frac{(2 \mathrm{l})^{2}}{2}=\theta^{\prime}(t) \cdot 1=v_{\mathrm{CM}}(t)
\end{aligned}
$$

Ou seja, a velocidade tangencial média dos trechos ao longo da ripa pode ser descrita pela velocidade tangencial na altura do centro de massa. Como a velocidade do perfil varia de zero (na altura do pivô) a $0,641 \mathrm{~m} / \mathrm{s}$, a velocidade média na posição do C.M. é metade, $0,321 \mathrm{~m} / \mathrm{s}$.

Substituindo-se na eq. 33, obtém-se a força de arrasto média na ripa, na primeira oscilação:

$$
\begin{array}{r}
\overline{F_{A R R}}=-\mathrm{C}_{x} \rho \bar{v}^{2} \mathrm{~A} / 2=-1,3.1,20 .(-0,0321)^{2} \\
.(0,473.0,010) / 2=-3,8.10^{-6} \mathrm{~N}
\end{array}
$$

Que corresponde a $3,8 \cdot 10^{-6} / 3,1 \cdot 10^{-3} \approx 0,1 \%$ da força de atrito. Isto mostra porque a envoltória do decaimento oscilatório da Figura 7 é linear, com total predominância do atrito seco.

\subsubsection{No pivô do DVD}

Como já foi verificado, o DVD também tem sua amplitude de oscilação reduzida linearmente a cada ciclo, devido ao atrito seco do eixo pivô com o orifício por onde atravessava. Os coeficientes angulares das retas nos máximos das oscilações (Figura 8) resultaram em -0,0370 e 0,0381 (média 0,0376). Adotando-se a média dos valores absolutos entre ambos e utilizando a eq. 21

$$
\begin{aligned}
\mu & =a T l /(4 r)=0,0376.0,606 .(0,120 / 2) /(4.0,001) \\
& =0,342
\end{aligned}
$$

onde $\ell=R_{1}$ é o diâmetro do DVD e $r$ o raio do eixo pivô e o valor do período $T$ obtido a partir da frequência experimental ajustada da equação (31): $(T=1 / f=$ $1 / 1,651=0,606 s)$.

Uma comparação com coeficientes de atrito estimados na literatura, plástico (orifício) vs. metal (eixo) mostra que o coeficiente de atrito dos materiais poliméricos

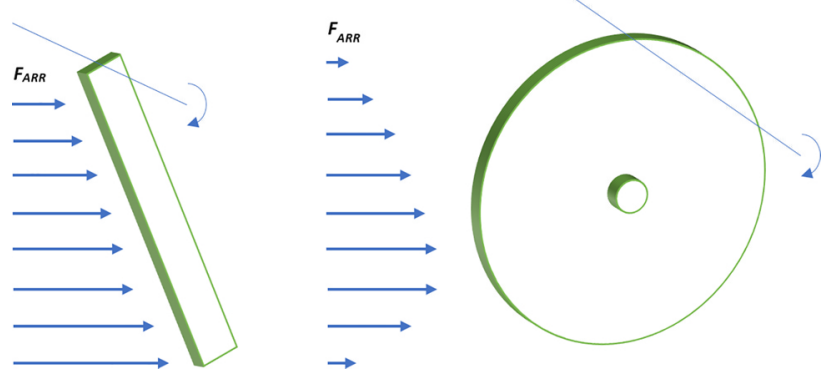

Figura 9: Forças de arrasto do ar ao longo dos corpos e áreas de seus perfis frontais ao arrasto encontra-se geralmente na faixa de 0,11 a 0,66 . O ABS, por exemplo, apresenta coeficiente de atrito entre 0,19 a 0,35. Algumas tabelas de coeficiente de atrito entre materiais estão, por exemplo, em [26], [27], [28], [29] e [30].

A força de atrito no pivô é calculada a partir da eq. 12. No ponto mais baixo da trajetória $(\theta=0)$,

$$
F_{\mathrm{AT}}=\mu m g=0,342.0,015.9,79=0,050 \mathrm{~N}
$$

Desprezou-se o atrito de arrasto aerodinâmico do DVD, calculado pela eq. 33. Mesmo assim, seu resultado foi estimado adiante. Este resultado é diferente da ripa, pelo fato de que a área frontal do perfil do DVD apresenta um coeficiente $C_{x}$ e não foi encontrado em tabelas valor para o caso dele girar em torno de um pivô na sua borda. Assim, considera-se que $C_{x}<2,0$ (tipicamente). A área da secção frontal ao movimento do DVD, não considerando que, devido à sua curvatura circular, não colide perpendicularmente com o ar ao longo da sua borda (Figura 9). Mesmo assim, considera-se para estimativa um pior caso, em que a secção frontal está perpendicular ao movimento e corresponde ao diâmetro do DVD vezes sua espessura 0,120 . 0,002 =2,4.10 ${ }^{-4} \mathrm{~m}^{2}$.

Analogamente à ripa, a velocidade tangencial média do perfil do DVD é descrita pela velocidade tangencial na altura do centro de massa. A velocidade do perfil na extremidade do DVD, com medição feita pela ferramenta do Tracker "medir declive" (na janela de análise de dados), foi obtida como valendo $0,38 \mathrm{~m} / \mathrm{s}$, no ponto mais baixo da trajetória.

Obtendo-se a força de arrasto média no DVD, na primeira oscilação:

$$
\begin{aligned}
& \overline{F_{A R R}}=\mathrm{C}_{x} \rho \bar{v}^{2} \mathrm{~A} / 2=2,0.1,20.0,038^{2} \\
& .(0,120.0,002) / 2=4,2.10^{-7} \mathrm{~N}
\end{aligned}
$$

Que corresponde à relação $F_{\mathrm{ARR}} / F_{\mathrm{AT}}=4,2.10^{-7} / 0,050 \approx$ $8.10^{-6}$ de fato desprezível. Isto mostra porque a envoltória do decaimento oscilatório da Figura 8 é linear (total predominância do atrito seco).

\subsection{Decaimento exponencial da oscilação no final do movimento do DVD}

Na Figura 8, nota-se que há um bom ajuste da envoltória linear sobre a curva de oscilação até o tempo de 9,0 s. No restante do movimento (detalhe na mesma figura), notase um decaimento amortecido. Procurou-se na literatura algum sistema oscilatório que exiba este comportamento espontaneamente (oscilação amortecida linearmente alterando seu regime para amortecimento viscoso), não tendo sido nada encontrado durante o tempo em que este trabalho foi desenvolvido. No caso do experimento aqui realizado, algum fator existente na montagem do sistema provocou esta situação, uma vez que era esperado o atrito seco retirar energia do sistema até seu movimento cessar completamente. 


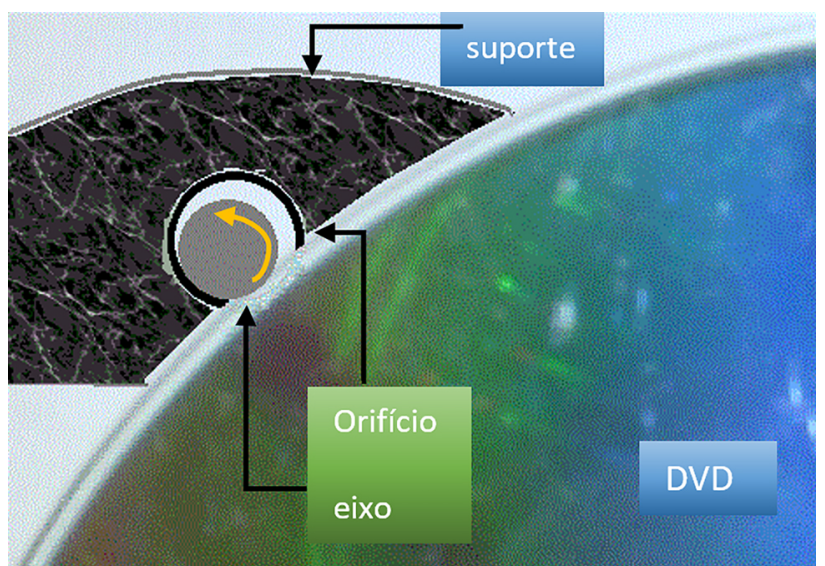

Figura 10: Detalhe esquemático do rolamento do eixo no interior do orifício

Analisando detalhadamente o sistema, observou-se uma folga entre o eixo que sustentava o DVD e o orifício por onde este eixo passava (Figura 6). Tal folga era suficiente para que, no final do movimento, houvesse um rolamento entre o eixo e a borda interna do orifício, para pequenas oscilações (detalhe na Figura 10). Até o instante de $9 \mathrm{~s}$, a amplitude de rotação e o peso do DVD não permitiam esse rolamento e o eixo deslizava circularmente no interior do orifício. Como o atrito de rolamento é muito menor que o de deslizamento (objeto deste estudo), após o tempo de 9s, a energia do sistema era essencialmente retirada pelo mínimo atrito com o ar (a despeito da seção de choque do perfil de DVD ser muito pequena e suas faces laterais bem lisas, resultando em um arrasto muito pequeno). Assim, no final do movimento, o pêndulo entrava em um regime de oscilação amortecida por atrito viscoso, sem o atrito eixo-orifício, explicando o decaimento exponencial final.

Com o auxílio do programa Tracker, esta parte do movimento, a partir de $9,1 \mathrm{~s}$, foi parametrizada com a equação 35 , baseada na equação 9 :

$$
\theta(t)=A \exp (-B(t-t 0)) \cos (2 \pi f .(t))+C
$$

em que o termo $\exp \left(-B\left(t-t_{0}\right)\right)$ parametriza o decaimento da curva de oscilação cossenoide. Os outros parâmetros são interpretados da mesma maneira que na eq. 30 e o resultado obtido foi:

$$
\begin{aligned}
\theta(t)= & 0,04176 \cdot \exp (-1,256(t-9,177)) \\
& \times \cos [2 \pi .1,659 .(t 9,177)]-1,576
\end{aligned}
$$

com desvio médio quadrático de 0,00057.

\subsubsection{Obtenção do coeficiente de amortecimento no regime de atrito viscoso}

Obtido o valor do momento de inércia dinâmico, torna-se possível calcular a constante de amortecimento rotacional deste movimento como (ver eq. 8)

$$
b=B \cdot 2 I_{P}=1,256.2 .8,14 \cdot 10^{-5}=2,04 \cdot 10^{-4} \mathrm{~kg} \cdot \mathrm{m}^{2} / \mathrm{s}
$$

A constante de amortecimento é muito pequena, mas diferente de zero, o que faz com que as frequências angulares harmônica não amortecida $\omega_{0}=8,489 \mathrm{rad} / \mathrm{s}$ e amortecida $\omega=\sqrt{\omega_{0}^{2}-B^{2}}=8,396 \mathrm{rad} / \mathrm{s}$, divirjam em cerca de $1,1 \%$. A amplitude da eq. 9, dada pelo termo $\theta_{0} e^{-b t /\left(2 I_{P}\right)}$, diminui gradualmente com o tempo e corresponde a envoltória da curva da Figura 8, no detalhe superior.

\section{Considerações Finais}

O uso do programa "Tracker" facilitou a tomada e análise dos dados experimentais, e seus resultados apresentaram grande concordância com os cálculos do momento de inércia obtido a partir das dimensões dos corpos utilizados. A disseminação de aparelhos portáteis tais como tablets e smartphones, com capacidade de filmagem, bem como a disponibilidade de computadores atualmente nas escolas e a gratuidade deste programa, permitem uma maior facilidade no aprendizado dos alunos no estudo de fenômenos físicos. Além disso, o "Tracker" é um ambiente em que integra o vídeo, a coleta de dados e os cálculos necessários para o problema em estudo, facilitando as análises dos parâmetros experimentais de movimentos.

Este modo de análise experimental também permitiu a comparação com os modelos teóricos, além de estimar o coeficiente de atrito com precisão de $1 \%$, o que, didaticamente, permite a comparação de um sistema real diante de um modelo de movimento e a estimativa dos desvios ocorridos.

No caso do DVD, comparando os resultados de 818 e 814 g.cm², observa-se um erro relativo de $0,5 \%$, que é menor que alguns resultados da literatura. Para a ripa, a diferença entre o cálculo teórico (geométrico) e o experimental foi de $3,5 \%$. Ainda no caso da ripa consideramos um experimento inédito, no sentido de gerar um atrito coulombiano em um pêndulo físico, com a ressalva de que não foi possível determinar a força de contato com a superfície lateral nas condições experimentais apresentadas.

A literatura apresenta inúmeros artigos de pêndulos simples rastreados por câmera, bem como trabalhos em que a massa pendular está imersa em líquido com o intuito de medir sua viscosidade. Há ainda outros trabalhos em que pêndulos simples metálicos são desacelerados por forças magnéticas. Optamos por não citar aqui tais trabalhos devido a estarem conceitualmente um pouco mais distantes de nossos experimentos.

A assunção do modelo de atrito coulombiano, isto é, independente da velocidade de escorregamento, se mostrou adequada para a análise dos experimentos aqui realizados, uma vez que a perda de energia observada foi praticamente constante durante todo o movimento. 


\section{Referências}

[1] F. S. d. Rocha, G. F. Marranghello e M. M. Lucchese, Revista De Enseñanza De La Física, 1 , 119-123 (2018).

[2] A. G. Lemes, F. D. Prado, D. Kiametis, A. S. Silveira, A. A. R. Coelho e C. Meza, em XVIII Congresso Brasileiro de Automática (Bonito, Mato Grosso do Sul, 2010).

[3] P. Olejnik e J. Awrejcewicz, Mechanical Systems and Signal Processing 98, 91 (2018).

[4] F. J. Arnold, R. Arthur, L. L. Bravo-Roger, M. S. Gonçalves e M. J. G. d. Oliveira, Revista Brasileira de Ensino de Física, 33, 4311 (2011).

[5] W. Bonventi Jr.e N. Aranha, Revista Brasileira de Ensino de Física, 237, 2504 (2015).

[6] A. Marchewka, D. S. Abbott e R. J. Beichner, American Journal of Physics, 72, 477 (2004).

[7] J. J. Crisco, J. Blume, E. Teeple, B. C. Fleming e G. D. Jay, Proceedings of the Institution of Mechanical Engineers, Part H: Journal of Engineering in Medicine, 221, 325 (2007).

[8] R. C. Hudson and C. R. Finfgeld, American Journal of Physics, 39, 568 (1971).

[9] J. Awrejcewicz and P. Olejnik, Transactions of the Applied Mechanics Reviews, 58, 389 (2005).

[10] https://www.physlets.org/tracker/. [Accessed oct 2019].

[11] F. P. Beer, E. R. Johnston Jr. e P. J. Cornwell, Mecânica Vetorial Para Engenheiros: Dinâmica, (RS: AMGH , Porto Alegre, 2012).

[12] J. L. Meriam e L. G. Kraige, Engineering Mechanics, 6 ed., John Wiley \& Sons, 328, (2002).

[13] A. Ruina e R. Pratap, Introduction to Statics and Dynamics (Oxford University Press, Oxford, 2002).

[14] R. C. Hibbeler, Engineering Mechanics, (Prentice Hall, Nova Jersey, 2007).

[15] R. W. Soutas-Little e B. Inman, Engineering Mechanics, Thomson, Boston (2008).

[16] D. Halliday, R. Resnick e J. Walker, Fundamentos de Física, (LTC, Rio de Janeiro, 2012).

[17] D. H. Young e R. A. Freedman, Física I, (Pearson Education do Brasil, São Paulo, 2008).

[18] G. Baker e J. Blackburn, The Pendulum: A Case Study in Physics, (Oxford University Press, Oxford, 2005).

[19] P. Grego, Dinâmica de um Autotensionador Mecânico Sujeito ao Atrito Seco. Dissertação de Mestrado, Faculdade de Engenharia Mecânica, Campinas, SP, (2015).

[20] L. Mezzalira, G. Moscati e J. M. Saffar, em ENQUALAB2006 - Congresso e Feira da Qualidade em Metrologia (São Paulo, 2006).

[21] https://www.geografos.com.br/cidades-sao-paulo/sorocaba.php, acessado em 19 setembro 2019.

[22] C. Pintão, M. S. Filho e W. F. Usida, Revista Brasileira de Ensino de Física 27, 237 (2005).

[23] T. Eadkhong, R. Rajsadorn, P. Jannual e S. Danworaphong, European Journal of Physics 33, 615 (2012).

[24] T. J. Bonagamba, E. Santoni, P. R. O. Lasso, C. B. Bretas e A. Gentil, Revista Brasileira de Ensino de Física 17, 133 (1995).

[25] F. Brunetti, Mecânica dos Fluidos, (Prentice Hall, São Paulo, 2005).

[26] http://structx.com/Material_Properties_005a.html, acessado em nov 2019.
[27] https://mechguru.com/machine-design/typicalcoefficient-of-friction-values-for-commonmaterials/, acessado em nov 2019.

[28] http://rommet.com/remco/misc/friction.htm, acessado em nov 2019.

[29] https://www.engineeringtoolbox.com/frictioncoefficients-d_778.html, acessado em nov 2019.

[30] A. S. Pouzada, E. C. Ferreira e A. J. Pontes, Polymer Testing 25, 1017 (2006). 